\title{
Delay discounting: Numerical estimation of gamification's impact on motivation and performance
}

\author{
Sophie Harvey ${ }^{1}$, Greg Jensen ${ }^{2,3,4}$, and Kristen G. Anderson ${ }^{2,5}$ \\ ${ }^{1}$ Dept. of Psychology, University of British Columbia \\ ${ }^{2}$ Dept. of Psychology, Reed College \\ ${ }^{3}$ Dept. of Neuroscience, Columbia University \\ ${ }^{4}$ Zuckerman Mind Brain Behavior Institute, Columbia University \\ ${ }^{5}$ Corresponding author (Kristen.Anderson@reed.edu)
}

\begin{abstract}
Gamification is the application of game design methods to a non-game context. To assess the impact of gamification on a common psychological task, we performed two experiments where the effects of gamification on the delay discounting task were evaluated. Experiment 1 compared three versions of the delay discounting task with differing levels of gamification and compared performance and task enjoyment across those variations. Participants found the task in the fully-gamified condition more enjoyable than the other conditions, although they also displayed small differences in task performance. Experiment 2 used two conditions, and participants once again enjoyed the gamified condition more than the control, despite performing comparably in both tasks. More sophisticated graphics and gameplay can provide a more enjoyable experience for participants without undermining delay discounting effects commonly reported in the literature.
\end{abstract}

Keywords: delay discounting, gamification, impulsivity, enjoyment

Delay discounting (DD) is a pattern of preference in which participants demonstrate that the value of a reward decreases as a function of how far into the future it is delayed. Studies using delay discounting paradigms have shown that both humans and animals tend to prefer smaller, more immediate rewards over larger, delayed rewards (Ainslie 1975; reviewed by da Matta et al. 2012). The delay discounting rate governs how rapidly a reward's value shrinks as its delay into the future grows. Thus, participants with a high rate of delay discounting will generally prefer small immediate rewards over much larger rewards delivered with even a moderate delay. On the other hand, a low delay discounting rate corresponds with a willingness to forego small immediate rewards in favor of larger delayed rewards, even if the day is considerable in length. Consequently, delay discounting has been used as a method of not only evaluating impulsive behavior but also as a model of decision making.

Tasks that measure delay discounting have been widely used to evaluate impulsivity and decision making in both monetary and non-monetary contexts. For instance, Bickel et al. (1999) used delay discounting to measure the comparative impulsivity of smokers, ex-smokers, and non-smokers. The authors used hypothetical monetary rewards for all groups in addition to cigarette rewards for the smoker group. They found that not only did smokers discount money to a greater extent than both ex-smokers and non-smokers, they also discounted cigarettes to a greater extent than the monetary rewards. Consequently, Bickel and colleagues concluded that those with drug dependence disorders are more likely to show a greater degree of discounting. In the context of video games, Weinstein et al. (2016) used delay discounting to examine internet gaming disorder (IGD) and impulse control. They divided participants into those who scored high on the Problematic Online Gaming Questionnaire (POGQ; Demetrovics et al., 2012) and those who scored low. Subsequently, all participants participated in a typical delay discounting task that involved choosing between a larger, delayed sum of money or a smaller, immediate sum of money. They found that the high POGQ group demonstrated significantly higher discounting than the low POGQ group, supporting the hypothesis the those with IGD show less impulse control than those without.

Unfortunately, the tasks used to measure delay discounting introduce a number of challenges. According to a recent meta-analysis, variations in experimental procedure substantially impact the estimated discounting rate (Rung et al., 2019). Across the ninety-two papers surveyed, the authors identified nine different manipulation types. Of these, ten studies focused on framing effects, which occur when descriptions of either time or the outcome described for a task are framed in terms of some hypothetical scenario. For instance, DeHart and Odum (2015) reported that participants discounted less steeply when the time delay was framed in calendar units (e.g., weeks, months, years) than when the delay was simply framed as days. Additionally, DeHart et al. (2018) examined how outcome framing could affect delay discounting and found that participants discounted less when the reward was framed as units of dollars (clear money) compared to when the reward was presented as units of 
handfuls of quarters (fuzzy money, p. 3). Similarly, Harman et al. (2020) provided monetary reward choices framed as either a quantity (an amount of dollar bills), volume (the height of a stack of dollar bills), or a duration (time spent in a hypothetical cash machine to collect dollar bills). They found that the duration condition had the steepest discounting curve while the quantity condition had the shallowest curve. These aforementioned studies demonstrate how sensitive delay discounting task design may be to manipulation. While increasing or decreasing a participant's discounting might be desirable in some cases, experimenters must be aware of the implications of seemingly minor framing changes when designing research protocols, particularly if they wish to compare discounting across studies. The purpose of this investigation was to examine motivational and performance impacts of gamification on delay discounting.

\section{Gamification}

Gamification is defined as "the use of game design elements in non-game contexts" (p. 10, Deterding et al., 2011). Certain game elements can be isolated and applied to non-gaming contexts to increase task motivation (Deterding et al., 2011), particularly where activities may seem boring or onerous. In the healthcare context, Garett and Young (2018) found that the most popular game elements were points, social interactions, leaderboards, and progress status. Many studies on the addition of these common gamification elements have proved fruitful in educational (Hakulinen et al., 2013) and employment contexts (Mitchell et al., 2020). However, changes to the task do not have to be substantive to potentially impact user interest; Lieberoth (2015) framification hypothesis states that simply framing a task as a game, even without having game mechanics, can still have an impact on engagement. If tasks can be successfully gamified to increase motivation without affecting the reliability and validity, rates of task completion may improve and provide better indices of performance in the laboratory or clinic. Several studies have already attempted to explore the impact of gamification on cognitive tasks. A 2016 review of gamification of cognitive tasks found that all studies that utilized a measure of intrinsic motivation in their experiment reported that gamification improved motivation on tasks when compared to non-gamified versions (Lumsden et al., 2016).

Birk et al. (2015) isolated four common psychological tasks (go/no-go, n-back, facial recognition, and ambiguous word) and gamified them by adding a premise and graphics. The go/no-go task is a commonly used inhibition task for measuring attention and reaction. In the control condition, participants were asked complete a traditional version of the go/no-go task by responding as fast as possible to circles displayed on a computer screen but not squares. The gamified condition had players aim and shoot blonde zombies but avoid moles wearing yellow hats. Birk and colleagues found no significant difference in player experiences between the two conditions; however, they found a strong decrease in player performance (measured by precision and accuracy) in the gamified condition. The author's suggested that the score difference was a result of the discrepancy between stimuli; telling a circle from a square is much easier than isolating the much more complex and detailed character designs present in their gamified condition (Birk et al., 2015). The experimenters found no differences in performance in the n-back and facial recognition task. However, Birk and colleagues also reported that participants demonstrated higher levels of enjoyment, autonomy, and immersion in the plain task, bringing forth the concept that gamification can actually negatively impact participant attitudes towards a gamified task.

In a subsequent exploration of gamifying cognitive tasks, Friehs et al. (2020) examined the stop-signal task, a measure of response inhibition, in two experiments. The first utilized a within-subjects design where participants played both a gamified and non-gamified version of the task. While the non-gamified version of the task played like a traditional version of the stop-signal task where participants must respond to arrows on a screen by pressing the corresponding arrow key (or withhold their response when a cue is played), the gamified version was based on an endless runner game. Endless runners are a genre of game where players run along an infinite path and must make quick decisions to dodge obstacles while attempting to run as far as possible. The gamified task had full 3D graphics, and task instructions were framed with a fantasy premise where the participants were informed that they were trapped in a magic forest by an evil witch and tasked with escaping. The authors found no differences in task performance between the two conditions. Additionally, the gamified version of the task was rated higher on the Interest/Enjoyment subscale of the Intrinsic Motivation Inventory (IMI; Ryan, 1982), and the order in which the tasks were completed had no impact on the results. In their second experiment, Friehs et al. utilized the same tasks and measures but applied a between-subjects design. While the experimenters again found no differences in task performance between the two groups, they found no difference in the Interest/Enjoyment subscale of the IMI between subjects. In other words, gamification only increased the enjoyment when participants could compare the two tasks.

Here, we focused on the delay discounting task as a target for gamification given its common usage in psychopathology research, validation against other indices of impulsivity, and potential amenity to gamification given the task parameters. Two experiments manipulated task framing and integration of game elements to determine whether gamification would result in enhanced motivation for participants without impacting task performance. For the current investigation, we applied a modest Bayesian analytic approach to fit the discounting function, appropriate for model comparison and detailed below. 
Fitting PSEs

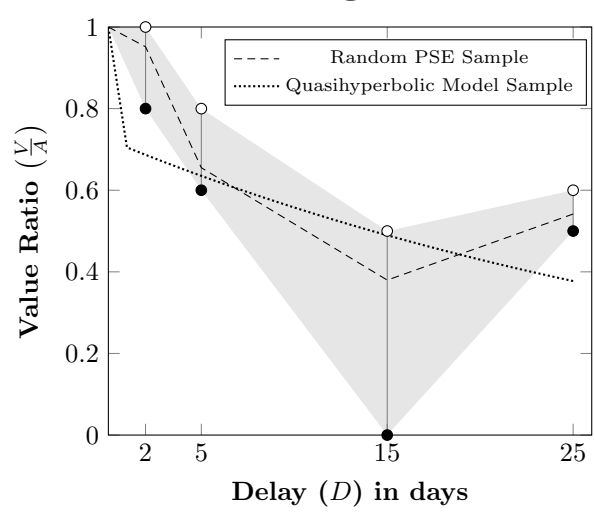

Nonparametric AUC

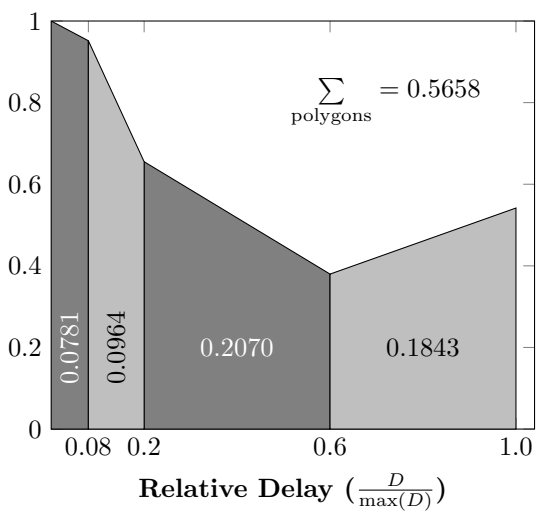

Model AUC

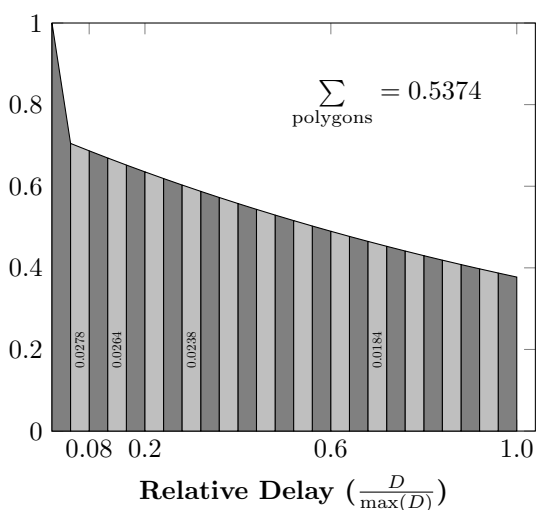

Figure 1. Example of how delay discounting behavior could be described for a hypothetical participant. (Left). Points represent offers for which the participant preferred a delayed reward worth $A$ (black points) and offers for which participant preferred an immediate reward worth $V$ (white points) at various delays. The participant's indifference point cannot be observed directly, as it is a latent variable lying somewhere between the black and white points at each delay. The dashed lines connect a possible set of values for each indifference point. Additionally, this participant's behavior is summarized by the quasihyperbolic model (dotted curve). (Middle). A nonparametric AUC, based on the indifference points plotted in the left panel. Although free of model assumptions, this description is dominated by its largest polygons, and thus is very sensitive to the spacing of the delays. (Right). A parametric AUC, based on the quasihyperbolic model plotted in the left panel. Since a parametric AUC can be divided into arbitrarily small polygons, it is much less sensitive to irregular spacing of delays.

\section{Analysis of a Gamified Delay Discounting Task}

Rather than being a self-reported characteristic, delay discounting is instead measured through revealed preference between pairs of offers (e.g., "Would you like \$1 now or \$10 in a week?"). Changes in the duration of the delay and the size of the reward change a participant's preference for the immediate reward. Between their smaller-sooner preference and their larger-later preference lies a hypothetical ratio of values where the effect of the delay balances out the size of the reward. This is known as the indifference point for a given delay (Reynolds and Schiffbauer, 2004). Figure 1 (Left) depicts an example in which immediate reward values $V$ are compared to delayed reward values $A$ as a series of ratios. In this example, the participant rejected the offers represented by the black points but accepted the offers represented by the white points. From this, we may infer that this participant's indifference point for each delay lies somewhere within the gray shaded region.

Standard practice is to fit quantitative models using parameters that minimize the residual variance of each PSE from the function, usually by successive approximation, without an explicit model that describes the distribution of the residuals. This approach has two limitations. The first is that minimizing residual variance implicitly assumes homogeneous Gaussian error, and this assumption is all but guaranteed to be violated by any curve approaching zero at the limit. The second, more substantial problem, is that indifference points are not directly observed. Instead, one of the trial ratios is nominated as being the 'most indifferent,' despite the participant having expressed a preference on that trial. Thus, minimizing residual variance via curve-fitting does not account for uncertainty about the true indifference point, and the observation nominated as its stand-in may not be an unbiased estimate of its true value.

As an exception to this approach, Vincent (2016) described a fully hierarchical Bayesian model for delay discounting that treats the indifference point as a latent variable and fits participant-level and population-level posterior parameter distributions simultaneously. The resulting model correctly balances all of the associated uncertainties and maximally extracts information from the available data. In principle, such a model makes valid (if uncertain) inferences about individual discounting patterns even if each participant was only offered a single ratio to consider at each delay, greatly reducing the number of responses needed from a participant. However, implementing this analysis is a considerable challenge for someone unfamiliar with this style of Bayesian modeling (Lee and Wagenmakers, 2013).

In this study, we used a more modest Bayesian approach which is hopefully more approachable and more readily adaptable by other authors, albeit less powerful than Vincent's. Our approach fits a discounting function for one participant at a time and makes the following assumptions:

1. Some parametric delay discounting function $f(D \mid \theta)$ exists that describes how a participant's indifference point varies with respect to delay. Given any delay $D$ and model parameter(s) $\theta$, an indifference point is predicted. These outputs take the form $\frac{V}{A}$, corresponding to the ratio of the immediate reward $V$ divided by the larger-later reward $A$. Since $V$ and $A$ are taken to be positive, this output can never go below zero.

2. Although $f(D \mid \theta)$ governs a participant's behavior in general, they also display variability trial by trial. As such, on 
any given trial, a participant's indifference point is given by $f(D \mid \theta) \cdot \epsilon$, where $\epsilon$ is presumed to follow a log-normal distribution (chosen because an indifference point should never have a value below zero).

3. Since an indifference point is not directly observable, it is presumed, at delay $D$, to fall between the largest value of $\frac{V}{A}$ for which the participant rejects $A$ and the smallest value for which they accept $A$.

4. All parameters (those governing the discounting function $\theta$, the residual error $\epsilon$, and the unknown true indifference points at each delay $D$ ) are given weakly informative priors.

A major advantage of this formulation is that any discounting function $f(D \mid \theta)$ can be used, provided the analyst sets appropriate prior probability distributions for any the parameters that govern the function. Since a full posterior distribution jointly estimates all parameters simultaneously, this approach is appropriate for model comparison should an analyst wish to compare multiple different discounting models. The complete model, as well as details on how to run it using the Stan programming language (Team, 2021), are provided in the electronic supplement.

In this study, we used the quasihypberbolic model (Laibson, 1997):

$$
E\left(\frac{V}{A}\right)=\beta \delta^{D} \text { when } D>0, E\left(\frac{V}{A}\right)=1.0 \text { when } D=0
$$

Here, $V$ is the size of the smaller, immediate reward, whereas A is the size of the larger, delayed reward whose delay D is measured in days. $\beta$ and $\delta$ are both free parameters, with $\beta$ acting similarly to an intercept while $\delta$ governs the speed with which the discounting curve shrinks. The dotted line Figure 1 (dotted) represents a possible quasihyperbolic discounting function for the depicted data. Using this framework, this approach allows foreach participant's overall discounting behavior can be described without sacrificing uncertainty about measurement error. These descriptions can then be used to make consistent comparisons within and between experiments as to the impact of gamification on delay discounting.

\section{EXPERIMENT 1}

Three versions of the DD task with differing levels of gamification (control, framing, and full game) were developed to evaluate how the original DD task compares to versions with different gamified elements. The framing condition was based upon Lieberoth (2015) framification hypothesis which states that simply framing a task as a game, even without having game mechanics, can still have an impact on engagement. Based on previous research (Lumsden et al., 2016; Friehs et al., 2020), we anticipated that both the full game and framing tasks would demonstrate higher levels of enjoyment and intrinsic motivation than the control condition with the core impulsivity estimates from the DD task remaining the same.

\section{Methods \\ Participants}

A total of 100 participants, aged 24 to 92 completed the experiment (two participants chose not to report their age), 32 who identified as women and 68 who identified as men were recruited from Amazon's MTurk. The racial makeup was predominantly White $(n=67)$, but also included Asian or Pacific Islanders $(n=18)$, Black/African Americans $(n=8)$, Latinx/Hispanics, $(n=4)$, Native Americans $(n=2)$, and one participant who identified as Other. The overall mean score on the Game Genre Questionnaire was 1.95 (meaning that most participants had some experience with video games), with the most frequently played genres being Shooters $(M=2.52, S D=1.06)$, Platformers $(M=2.47, S D=0.94)$, and Action Role Playing Games $(M=2.43, S D=1.09)$.

\section{Measures}

Demographics. Participants completed a demographics survey that included questions about age, sex (i.e., Male, Female, Other, Prefer not to answer), and ethnicity (Asian or Pacific Islander, Black/African American, Caucasian/White, Latinx/Hispanic, Other, Prefer not to answer).

Game Playing. The Game Genre Questionnaire (GGQ; Israel, 2017) is an unpublished 32-item self-report scale evaluating video game engagement across genres. Participants were provided with a list of game genres (and example games of each genre) and asked to respond how often they play each type of game on a scale of Never, Seldom, Occasionally, and Frequently (coded as 1, 2, 3, and 4 respectively). This measure was successfully used in a small sample of undergraduates in previous gaming research and was included here to assess participants' familiarity and experience with interactive games.

Impulsivity. The UPPS-P Impulsive Behavior Scale (Cyders et al., 2007) is a 59-item self-report scale developed to assess impulsivity along multiple dimensions: sensation seeking $(\alpha=.90)$, the tendency to seek out and enjoy novel experiences, lack of premeditation $(\alpha=.88)$, the inclination to act without considering the risks or consequences, lack of perseverance $(\alpha=$ 

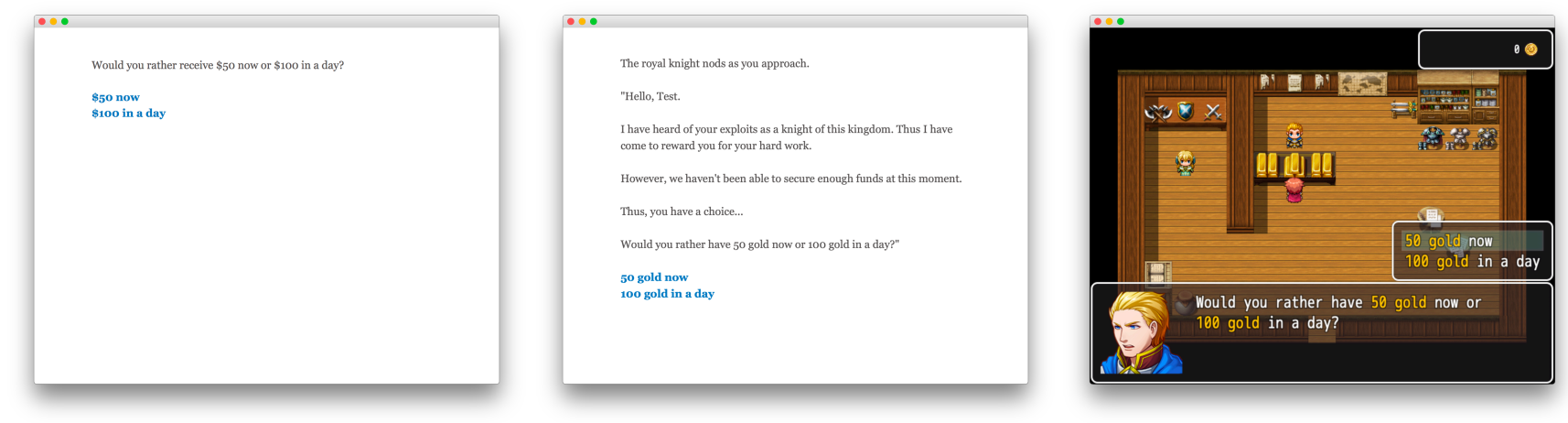

Figure 2. Example screens from the Control condition (Left), the Framing condition (Middle), and the Gamified condition (Right).

.88), the inability to remain focused on a given task, negative urgency $(\alpha=.91)$, the tendency to engage in risky behaviors when under negative emotions, and positive urgency $(\alpha=.95)$, the tendency to engage in risk behaviors when under positive emotions. The UPPS-P has been validated in adult populations across genders (Cyders, 2013) and showed excellent reliability in this sample.

Enjoyment. The Task Evaluation Questionnaire is a 22-item subscale of the Intrinsic Motivation Inventory (IMI; Ryan, 1982), a self-report questionnaire designed to assess a participant's experience in a target activity and asked to rate how true a statement is for them on a scale from 1 (not true at all) to 7 (very true). Four pertinent subscales assess aspects of the task including interest/enjoyment $(\alpha=.95)$, perceived competence $(\alpha=.82)$, perceived choice $(\alpha=.85)$, and pressure/tension $(\alpha$ $=.79$ ). The IMI has shown strong reliability and validity in adult samples (McAuley et al., 1989) and demonstrated good to excellent internal consistency in this sample as seen above.

DD Task. The task required participants to choose between two hypothetical amounts of currency between now and across four time periods: a day, a week, a month, and three months (i.e., "Would you rather receive $\$ 50$ now or $\$ 100$ in a day?"). The control condition replicated a typical DD study where participants are asked to choose between a hypothetical smaller, immediate sum of money or a delayed, larger sum of money. The framing condition utilized the same core narrative as the control condition but was framed with an added fantasy storyline where the player is given the role of a knight who must obtain money (gold) for their family by interacting with another character. Additional dialogue options were added such as conversing with another character and examining a room. Participants entered their name, later referenced throughout the task. In the gamified condition, the participants entered their name in addition to choosing between two avatars. The narrative and decisions were the same as the framing condition, but with the added ability to use the avatar to move and interact with objects and a non-player character who presented the DD task. A small window containing the participant's current amount of gold was added to the top right corner of the screen. The core DD procedure remained the same across conditions. The participants were first asked if they would rather have $\$ 50$ (or 50 gold in the framing and full game condition) now or $\$ 100$ in a day. If the participant selected $\$ 50$, this portion of the task ended, and the participant moved onto the next section. If the participant selected $\$ 100$, they were then asked if they would rather have $\$ 75$ now or $\$ 100$ in a day. This process continued with the immediate option increasing to $\$ 88, \$ 94$, and finally $\$ 97$. The procedure was again repeated with a delay of one week, one month, and three months.

Both the control condition and the framing condition were programed in Twine version 2.1.2 (Cox et al., 2021), an open-source framework for constructing text-based interactive stories. The full game was constructed in RPG Maker MV (Kadokawa Corporation, 2015), a program for creating two-dimensional role-playing games.

DD Measurement. Despite unambiguous evidence that individuals differ consistently with respect to delay discounting in general (Odum, 2011), no consensus has emerged as to which mathematical function is most appropriate to describe how the indifference point changes as a function of delay. For example, Franck et al. (2015) compared five different models, all non-linear and each having one or two parameters governing its shape. Wulff and van den Bos (2018) compared eight such models. This glut of models, from which no clear winner has emerged, has made it increasingly difficult to compare discounting results across the literature.

One approach is to describe delay discounting non-parametrically. Rather than trying to fit a curve, one can linearly interpolate between the indifference points at each delay and calculate the "area under the curve" (or AUC) of the resulting trapezoids (Myerson et al., 2001). Figure 1 (Center) selects indifference points from their possible ranges in Figure 1 (Left), and the area of each segment computed using relative delay. Given $n$ delays and assuming no discounting at time zero (that is, $D_{0}=0.0$ and $\frac{V_{0}}{A_{0}}=1.0$ ), the following sum gives the nonparametric AUC: 


$$
A U C=\sum_{i \in\{1: n\}}\left[\left(x_{i}-x_{i-1}\right) \frac{y_{i-1}+y_{i}}{2}\right] \text { where } x_{i}=\frac{D_{i}}{D_{n}} \text { and } y_{i}=\frac{V_{i}}{A_{i}}
$$

Since the delays are also relative, the resulting AUC has some value between 0.0 and 1.0. This allows comparison of any discounting effect to any other discounting effect, across studies and without relying on a specific model, at least out to the shortest common value of $D_{n}$.

However, this nonparametric approach has a number of important limitations. One of the main criteria for choosing a nonparametric measure is that it is robust (Büning, 2000); i.e., that it sacrifices some sensitivity to information in order to reduce the influence of irrelevant information. The median, for example, is robust because it is not influenced by extreme (and thus potentially mismeasured) outliers. For AUC to be robust, it should not depend too strongly on the specific set of delays in any given study. This is unfortunately not the case: the nonparametric AUC's sum is dominated by whichever trapezoids span the largest gaps between delays (Borges et al., 2016). Since most delay discounting studies use wider gaps between later delays, participant preferences measured at short intervals are inconsequential for most datasets.

Nonparametric AUC also has no way of accounting for measurement error. Since indifference points are not directly observable, an empirical approach is to offer the participant a large enough number of choices for the indifference point to get pinched into a narrow range, then pretend any remaining residual error does not exist. When participant responses are noisy, the nonparametric AUC is very sensitive to outliers, especially at long delays.

We selected a parametric approach to the AUC (Gilroy and Hantula, 2018). A robust theory of delay discounting requires using parametric models, but differing models cannot be compared in terms of their parameters. Such comparisons become possible if a parametric "model AUC" calculates the area under the formal model's discounting curve out to $D_{n}$. This does not require calculus; instead, one can calculate the value of the discounting function for small, uniformly spaced intervals and then Equation 2 to compute the sum over the resulting trapezoids. With finer slices, the sum converges on the true integral. Figure 1 (Right) depicts this approach as it applies to the model fit included in Figure 1 (Left).

\section{Procedure}

All procedures were approved by the Institutional Review Board of Reed College to assure ethical treatment of participants. Participants were recruited through an advertisement posted on MTurk. After obtaining consent, participants completed demographic items, the Game Genre Questionnaire, and the UPPS-P. Participants were randomly assigned to complete one of three tasks: control $(n=35)$, framing $(n=26)$, or gamified $(n=39)$. Subsequent to finishing the DD procedure, participants completed the IMI and compensated $\$ 1.00$ for their time.

\section{Results}

A bootstrapping analysis of the GGQ and the UPPS-P pre-task surveys, corrected for multiple comparisons using the Hommel procedure (Hommel, 1988), did not yield any significant differences between conditions, with one exception: participants in the Gamified condition scored significantly higher on the sensation seeking items of the UPPS-P than did participants in the Framing condition. On this basis, we concluded that participants were fairly well distributed across conditions. This analysis and the corresponding plot of the data are included in the electronic supplement.

Figure 3 (Top Row) plots the mean posterior quasihyperbolic discounting function for each participant in each of the three conditions as a function of the V/A ratio. Additionally, the overall mean discounting function across participants is plotted using heavy black lines, as are the mean estimated indifference points across participants. Participants displayed very similar discounting rates $\delta$ across conditions, but differed in their intercept parameters $\beta$, with the highest intercept (and thus least discounting) in the Control condition and the lowest intercept (and thus most discounting) in the Gamified condition.

Discounting was then characterized using a model AUC measure (using Equation 2 to numerically integrate the quasihyperbolic model), as depicted in Figure 3 (Bottom Left), participants discounted significantly more on average in the Gamified condition than the Control condition, after correcting for multiple comparisons using the Hommel procedure. When examining the model AUCs of individual participants (Figure 3, Bottom Right), this difference appears to mainly be driven by a larger proportion of participants who discounted heavily, rather than by a systematic shift downward of all participants. Only $23 \%$ (8/35) of Control condition participants had mean estimated AUCs less than 0.5, as compared to 31\% (8/26) of Framing condition participants and 44\% (17/39) of Gamified condition participants.

In order to understand participant motivation during and after the task, we used a general linear model to predict the four components of the IMI as a function of task condition and each participant's model AUC. The intercepts of this model are plotted in Figure 4 (Left). After correcting for multiple comparisons using the Hommel procedure, condition did not result in different estimated intercepts in Perceived Competence, Perceived Choice, or Pressure/Tension. However, the Gamified condition had a significantly higher level of Interest/Enjoyment than either the Control condition or the Framing condition. Figure 4 (Right) 

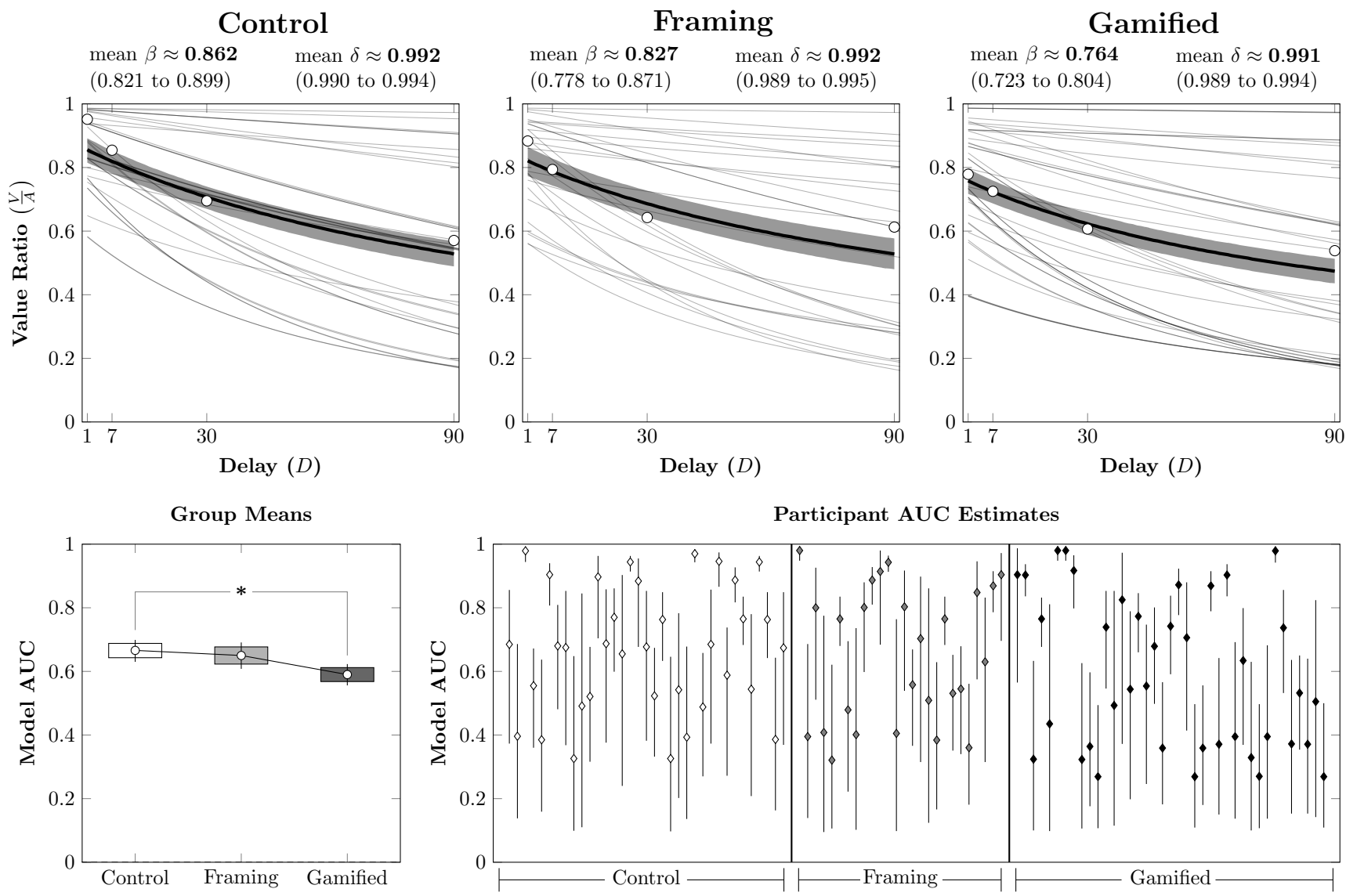

Figure 3. Discounting behavior across participants in Experiment 1. Error bars represent the $95 \%$ credible interval for the mean, while boxes represent the $80 \%$ credible interval. (*) represents a posterior distribution comparison that excludes zero from the $95 \%$ credible interval. (Top Row). Posterior means for each participant's estimated behavior, based on the quasihyperbolic discounting model in each of the three conditions. Thin gray lines represent participant estimates, whereas the thick black line represents the mean across that condition, with shading depicting the $95 \%$ credible interval of that mean. Also plotted are the empirical means across participants for the estimated indifference point at each delay. (Bottom Left). Mean parametric AUC across participants for each condition. (Bottom Right). Estimated parametric model AUC for each participant, based on the posterior parameter estimates of the quasihyperbolic distribution

depicts the estimated impact of participant AUC on each of these four components. Of these, only Interest/Enjoyment varied significantly as a function of AUC. This suggests that participants who derived greater Interest/Enjoyment from the task also displayed lower model AUCs. Thus, high interest was associated with stronger discounting.

\section{Experiment 1 Discussion}

We examined two primary effects: 1) whether gamification affects enjoyment of the core task, and 2) whether gamification impacts DD scores. On average, participants discounted more heavily in the Gamified condition relative to the Control and Framing conditions, seemingly driven by a greater proportion of individuals discounting more heavily in that condition relative to the others. Interestingly, the Gamified condition engendered the highest interest/enjoyment and varied as a function of participants' AUC, such that the greater enjoyment/interest someone reported was associated with greater discounting. Experiment 2 was designed to better address questions as to who might be more affected by gamification and in what DD context, as well as remediate design concerns identified after completion of the first study.

\section{EXPERIMENT 2}

Experiment 2 was designed to improve experimental control from the first experiment. Due to the gamified nature of the full game condition in Experiment 1, certain game design elements were implemented that were not feasible in the non-game conditions, leading to potential confounds. The most distinguishing elements were the inclusion of a player avatar (a digital representation of the player) as well as player-controlled movement. In Experiment 2, we decided to remove the avatar entirely as well as the ability to move volitionally. We looked towards the design of visual novels for gamification, a medium 

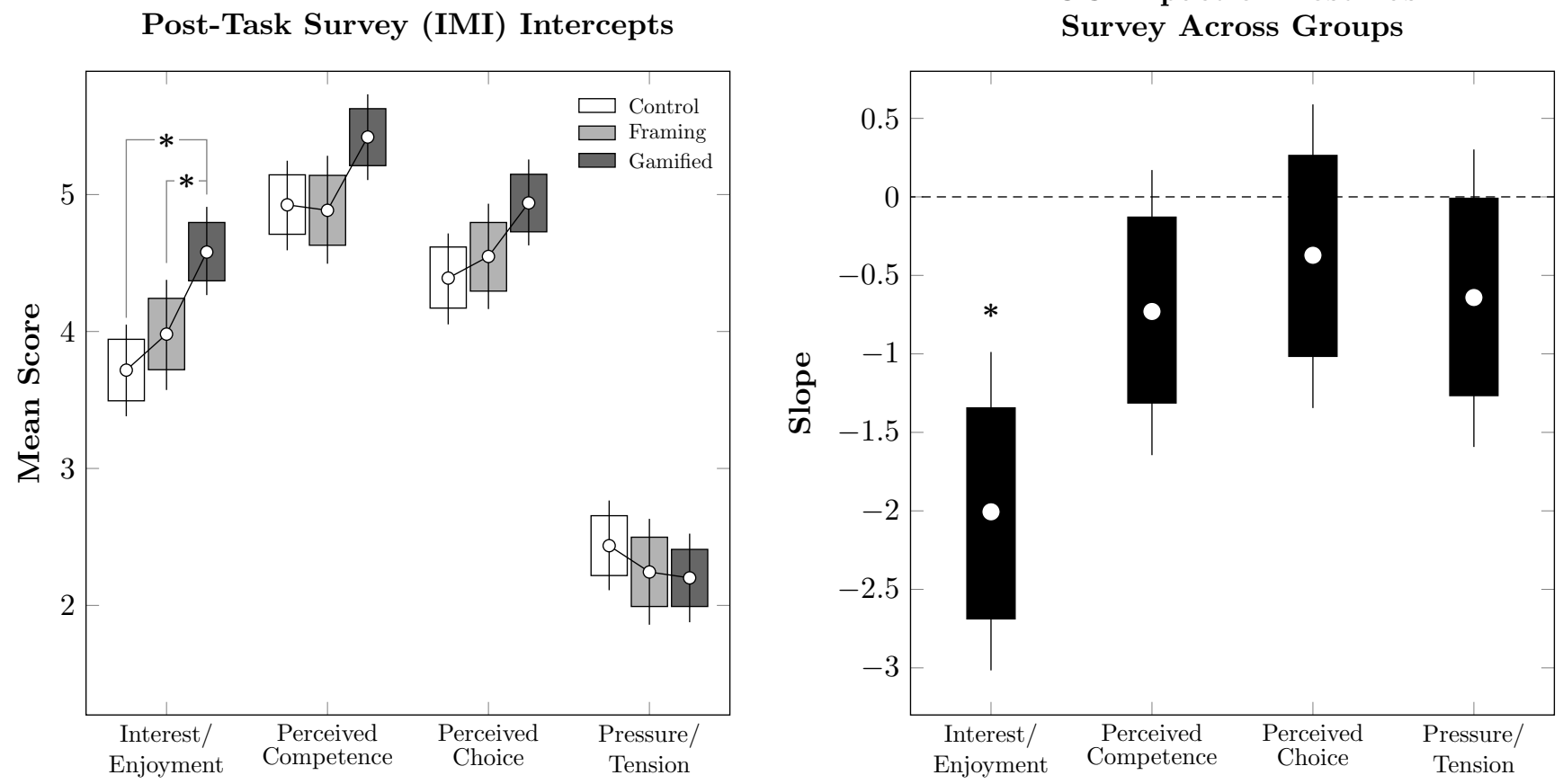

Figure 4. Impact of delay discounting task participation on post-task IMI scores in Experiment 1. Error bars represent the $95 \%$ credible interval for the mean, while boxes represent the $80 \%$ credible interval. $\left(^{*}\right)$ represents a posterior distribution comparison that excludes zero from the $95 \%$ credible interval. (Left). Mean scores for each of the IMI's four components in each of the conditions, after controlling for a participant's model AUC. (Right). Mean slopes describing how each of the IMI's four components change as a function of a participant's model AUC.

that combines interactive fiction (typically represented by branching narratives) with visuals such as character sprites and backgrounds (Cavallaro, 2009). It is not uncommon for the main character to not be seen in visual novels, giving the illusion that the game is being presented in a first-person format. Several studies have previously examined the use of visual novels in an experimental setting. For instance, Faizal (2016) demonstrated that students who used a visual novel to study English scored significantly higher on academic achievement tests than those in the control group. Thus, it was hoped that combining the narrative present in the first study with the framework of a visual novel would provide a more enjoyable experience than the basic control task without potential confounds associated with self-referent avatars and movement.

While participants were not able to predict how long the subsequent delays would be in Experiment 1, they may have discerned a pattern in the amount of money presented as it always followed the same sequence. If participants became aware of the pattern, they could even predict what the final offer of each time delay was going to be, meaning they could always choose to wait for that final offer if they wished. We modified the randomization of the DD time delays and corresponding payouts and included additional time delays (three and five days) to test delay distributions that were more consistent with other studies within the literature (Rachlin et al., 1991; Borges et al., 2016). We also improved our assessment of gaming history by using a validated measure of gaming experience, the Game Preference Questionnaire (GPC; Manero et al., 2016), allowing us to better model how individual experience with gaming might relate to susceptibility to gamification and discounting on the task.

\section{Methods \\ Participants}

As in the previous study, all participants were recruited through MTurk. One hundred eighty-nine participants, ranging from ages 22 to 72, completed the study. The majority of the participants fell between the ages of 31 to $40(n=80)$ and over half identified as male $(n=108)$. The sample was also comprised of 79 women and well as one participant who identified as Other in addition to one participant who chose not to answer. While the sample was predominantly White $(n=116)$, it also included Asian or Pacific Islanders $(n=57)$, Black/African Americans $(n=9)$, Latinx/Hispanics $(n=2)$, participants who identified as Other $(n=2)$, and participants who preferred not to answer $(n=3)$.

\section{Measures}

All self-report measures described for Experiment 1 were replicated in Experiment 2 ( $\alpha$ ranging from .73 for IMI Pressure/Tension to .97 for Positive Urgency), with the exception of the GGQ that was replaced by the GPQ. This measure is a 

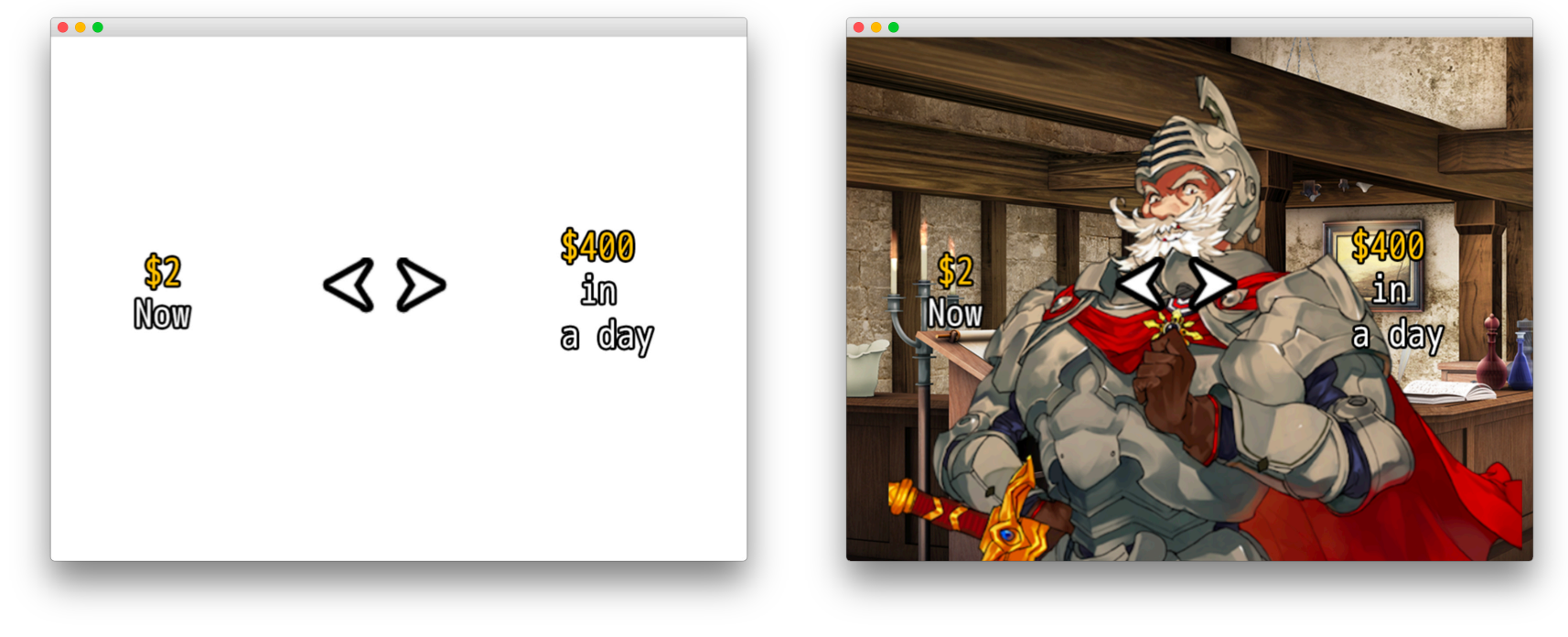

Figure 5. Example screens from the Control condition (Left) and the Gamified condition (Right).

10-item self-report scale utilized to classify gamers based on their game preferences and habits. The measure then provides a formula that classifies participants as best matching one of four categories: casual gamers (CG), well-rounded gamers (WRG), hardcore gamers (HG), and non-gamers (NonG) (Manero et al., 2016).

The DD procedure was expanded to five time delay sections: one day, three days, five days, one months, and six months, resulting in four sets of relative DD pairs (\$5-\$1000, \$400-\$800, \$3-\$600, and \$2-\$400). A randomized set of DD choices was chosen from the aforementioned pairs for each time delay, and the pairs were presented on the left and right side of the screen (also randomized), with unelectable arrows placed in-between. To be more analogous to the standard DD administration, both conditions used the word "dollars" rather than using the abstract "gold" used in Experiment 1 to reduce potential fuzziness.

\section{Procedure}

Participants were randomly assigned to one of two conditions: Control $(n=94)$ or Gamified $(n=94)$. The control version provided instructions and then asked the participant which amount of currency (represented by dollars) they would rather have. In contrast to the first experiment's gamified task where participants were given a choice of avatar and allowed to explore, this gamified task emulated a visual novel. Consequently, while the player was able to interact with the world via menu-based commands, there was no avatar to manipulate. The narrative in the gamified version was roughly the same as that presented in the first study, although with a few additions to compensate for the extra time delay. Participants completed the entirety of the study online through SurveyMonkey.

\section{Results}

Analysis of the GPQ assigned each participant to one of four categories of gamer. Our sample achieved reasonably good coverage of these four types, with double-digit counts of each category in our two task conditions: casual gamer cluster $(n=$ $61)$, well-rounded gamer $(n=52)$, hardcore gamer $(n=34)$, and non-gamer $(n=42)$. Additionally, an analysis of the UPPS-P yielded no significant differences between task conditions; details are included in the electronic supplement.

Figure 6 (Top Left) depicts the mean quasihyperbolic discounting functions for each of the 92 participants in the Control condition, while Figure 6 (Top Center) depicts the discounting functions for the 94 participants in the Gamified condition. Both conditions displayed similarly broad ranges of individual discounting patterns, but the conditions nevertheless yielded nearly identical mean discounting parameters.

Figure 6 (Top Right) shows the bootstrapped mean model AUC for participants, as a function of condition, GPC category, and their intersection. After controlling for multiple comparisons using the Hommel procedure, there was no overall difference in discounting between the Control and Gamified conditions. However, there were substantial differences in discounting as a function of gamer category. Well-rounded gamers discounted the most, followed by casual and hardcore gamers, whereas non-gamers discounted the least. Only casual gamers showed a difference in discounting as a function of the task, doing so more in the Control condition than in the Gamified condition. That said, every gamer category in every condition displayed a range of discounting behaviors, as depicted by the participant estimated model AUCs in Figure 6 (Bottom).

As in Experiment 1, a multivariate regression simultaneously evaluated the four components of the IMI with respect to condition and participant AUC. Additionally, the four gamer categories were included in this analysis. The intercepts of this 

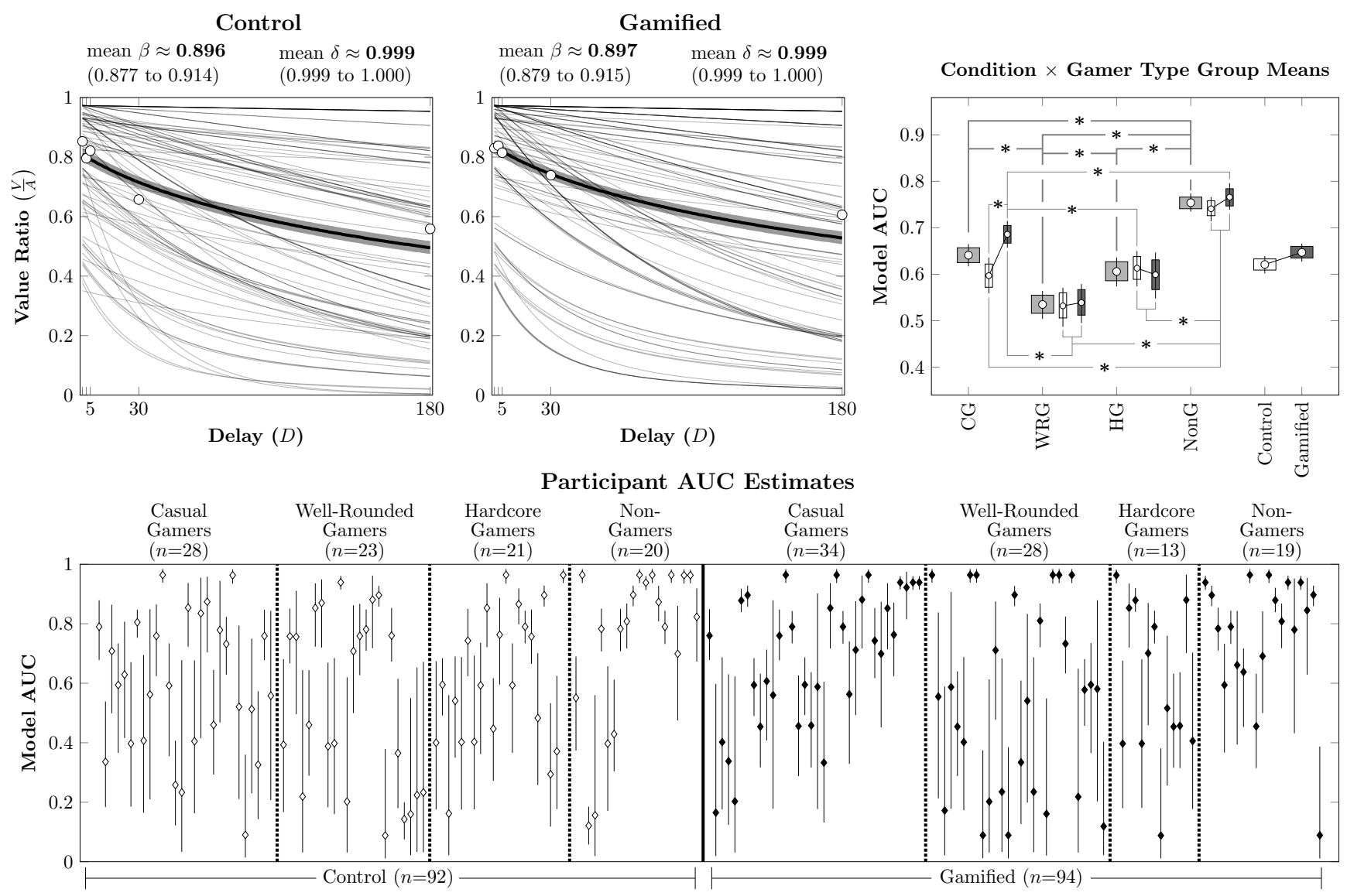

Figure 6. Discounting behavior across participants in Experiment 2. Error bars represent the $95 \%$ credible interval for the mean, while boxes represent the $80 \%$ credible interval. (*) represents a posterior distribution comparison that excludes zero from the $95 \%$ credible interval. (Top Left \& Center). Posterior means for each participant's estimated behavior, based on the quasihyperbolic discounting model in each of the two conditions. Thin gray lines represent participant estimates, whereas the thick black line represents the mean across that condition, with shading depicting the $95 \%$ credible interval of that mean. Also plotted are the empirical means across participants for the estimated indifference point at each delay. (Top Right). Mean parametric AUC across participants for each condition and subdivided according to the gamer type that best describes participants. (Bottom). Estimated parametric model AUC for each participant, based on the posterior parameter estimates of the quasihyperbolic distribution.

model are depicted in Figure 7 (Left \& Center). Taken overall, participants rated the Gamified condition higher than the Control condition for Interest/Enjoyment and Perceived Choice. With regards to the intersection of condition and gamer category, the most dramatic effect was that non-gamers in the Control condition reported significantly lower Interest/Enjoyment than any other pairing (after correcting for multiple comparisons using the Hommel procedure), whereas non-gamers in the Gamified condition enjoyed the task about as much as all other participants.

Participant model AUC was significantly predictive of Interest/Enjoyment and Pressure/Tension. As such, participants who showed stronger discounting (and thus lower AUC scores) reported higher values than those who did not discount as much.

\section{Experiment 2 Discussion}

The primary aim of Experiment 2 was to replicate the result of increased enjoyment within Experiment 1 without impacting participant's performance on the DD task. While the Gamified condition yielded higher levels of interest/enjoyment (as well as perceived choice), a complex interaction with GPQ's four categories of game player was also observed that resulted in substantial differences in both discounting and interest/enjoyment. Setting this complexity aside, however, participants nevertheless appeared to be more engaged with the task in the Gamified condition, without this coming at the expense of their discounting performance differing appreciably from the Control condition.

\section{GENERAL DISCUSSION}

The purpose of this investigation was to determine whether gamification of a DD task would impact task motivation and performance. Experiment 1 indicated that interest/enjoyment for the task improved when full game elements were included; 

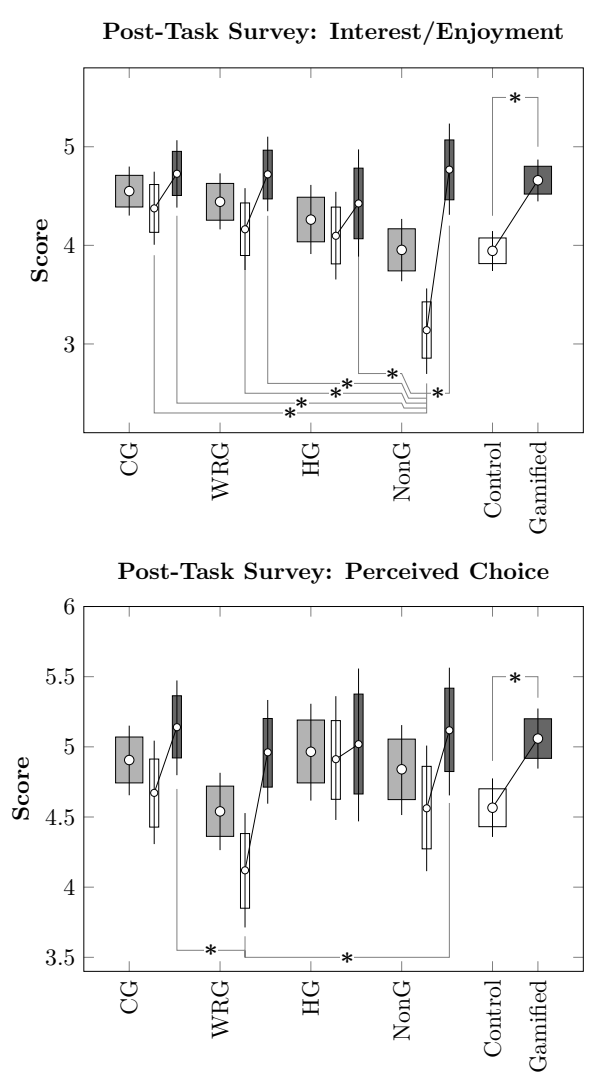

Post-Task Survey: Perceived Competence

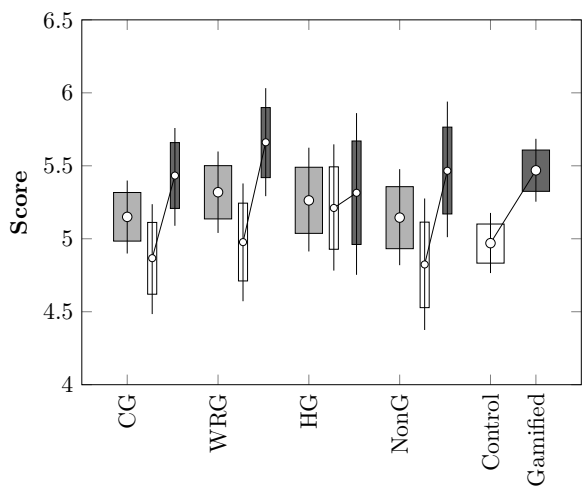

Post-Task Survey: Pressure/Tension

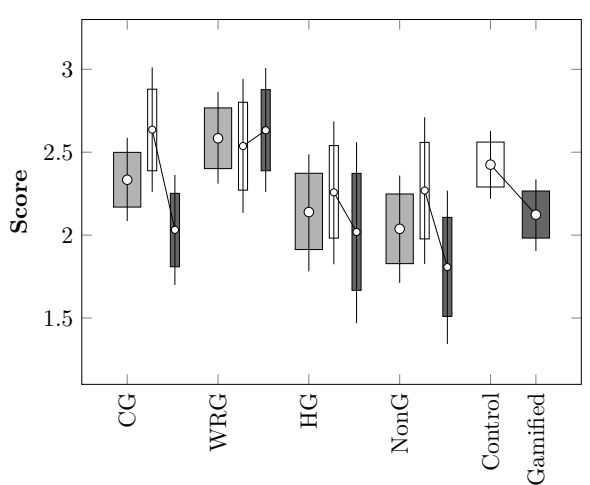

AUC Impact on Post-Task

Survey Across Groups

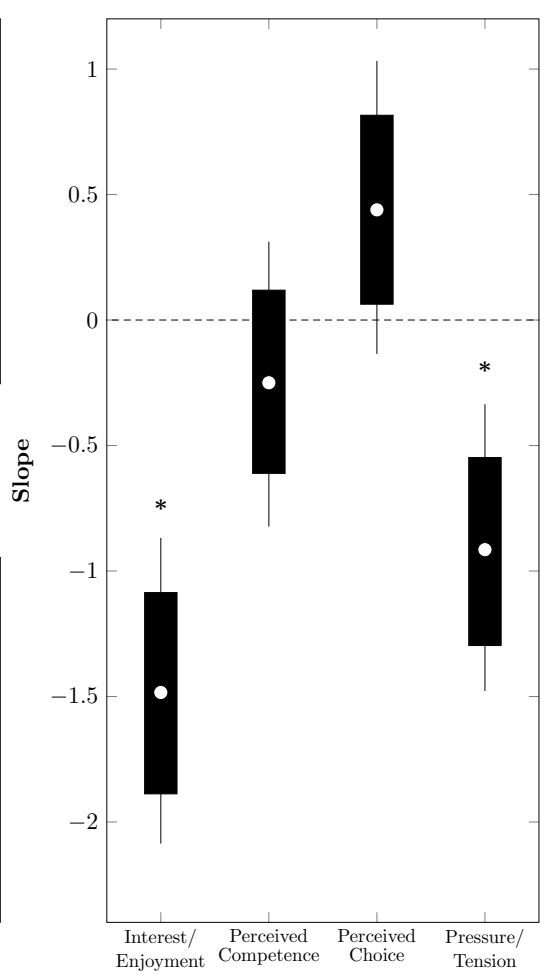

Figure 7. Impact of delay discounting task participation on post-task IMI scores in Experiment 2. Error bars represent the $95 \%$ credible interval for the mean, while boxes represent the $80 \%$ credible interval. (*) represents a posterior distribution comparison that excludes zero from the $95 \%$ credible interval. (Left). Mean scores for each of the IMI's four components in each of the conditions and subdivided according to the gamer type that best describes participants, after controlling for a participant's model AUC. (Right). Mean slopes describing how each of the IMI's four components change as a function of a participant's model AUC.

however, gamification increased discounting in that condition as compared with the control condition, albeit to a limited degree. Using a more modest gamification strategy in Experiment 2, DD performance was uniform across conditions with greater interest/enjoyment and perceived choice reported in the gamified condition relative to control. However, these findings masked unexpected and complex differences when gaming history was considered. Participants without gaming experience had the lowest interest and enjoyment in the control condition than other gamer types but were indistinguishable in the gamified condition. Participants who discounted the most, or the most impulsive, also endorsed greater motivation in the domains of interest/enjoyment and pressure/tension than those who discounted less.

These findings add to the existing literature on framing, gamification, and the implementation of the DD task. Our findings support the framification hypothesis (Lieberoth, 2015) as we demonstrated that relatively modest changes to how the task is framed, such as presenting the choices in the style of a visual novel, are sufficient to increase enjoyment of the DD task. While more complicated aspects of gamification, like an avatar whose movement is under player control, also improve motivation, caution should be exercised to ensure that such additions do not substantially change task performance, potentially limiting the validity of the task (see Birk et al., 2015). Our gamification of the DD task resulted in little to no impact on task performance, a similar result to what was reported in the review by Lumsden et al. (2016). Our findings regarding the interaction of gaming history with motivation and discounting also has implications for the broader literature on media consumption and gaming. Additionally, the division of participants into different varieties of game player in the second experiment it consistent with the findings of Weinstein et al. (2016), suggesting that individual preferences for certain forms of media may be predictive of their overall impulsivity, with "well-rounded gamers" showing the strongest propensity for discounting and "non-gamers" displaying the weakest propensity for doing so.

Our use of a modest and adaptable Bayesian modeling framework expands the reach of these techniques in the DD literature. Unlike traditional curve-fitting methods, our model recognizes and accommodates measurement error, allowing us to treat indifference points as latent variables. This approach allows unbiased estimates of discounting with appropriate uncertainty, 
even when the number of observations is small. Although we used a quasihyperbolic discounting model, this approach can be used with any parametric discounting function. Similarly, our use of parametric AUCs facilitates the comparison of results across studies and across theoretical divides, in a manner that comparing model parameters does not. As we argue above, this approach is a better compromise than the original proposed framework of "nonparametric" AUC measures, as it works in harmony with measurement uncertainty, rather than ignoring its contribution to the resulting description of discounting.

Additional limitations of this work must be noted. While our use of an online data collection format allowed for recruitment of a more diverse age range than typically found in laboratory studies in the area, it does incorporate variability in the administration of the tasks that cannot be assessed. Participants recruited online may differ in terms of their familiarity with tasks presented and characteristics germane to this investigation (e.g., impulsivity, familiarity of games) than those typically used in DD studies. However, Schluter et al. (2018) found that participants recruited through MTurk performance on the DD task was both logically consistent and showed within task reliability with the similarly administered Balloon Analogue Risk Task. While the ages represented in the sample were broader than typical undergraduate samples, participants were predominantly white, a continued issue with psychological research.

DD tasks have a tendency to be long, repetitive, and uninteresting (Robles and Vargas, 2007), factors that may negatively impact data quality DeRight and Jorgensen (2015). Gamification might be an effective strategy to combat motivational issues among participants on cognitive tasks, like DD. The definition of gamification given by Deterding et al. (2011) involves isolating singular mechanics of games and incorporating them into non-game contexts, a definition criticized for not fully encapsulating why people enjoy games (Werbach, 2014). Players do not return to games simply because of a collection of individual elements, like scores or leaderboards; games are complex systems with which players interact. Our findings support the complexity of the task-user interaction in the DD context and the importance of framing in how scientists construct cognitive tasks. How tasks are framed, whether that be through gamification or not, has the potential to improve the externally validity of our laboratory procedures and participant motivation to optimize task performance. That said, caution must be exerted to maintain the key elements of the tasks and data analysis to allow comparison across studies.

\section{Acknowledgments}

Many thanks to Carol Franceschini, Ph.D. for her input on the design of Study 2. Additional thanks to Mario Ferrari, M.A. for his assistance in data analysis on early drafts of this manuscript.

\section{Funding}

This work was performed with the support and funding of the Reed College Summer Research Award.

\section{Author Contributions}

The study was designed by SH \& KGA. Data were collected by SH. Data were analyzed and the paper was written by SH, GJ, \& KGA.

\section{REFERENCES}

Ainslie, G. (1975). Specious reward: A behavioral theory of impulsiveness and impulse control. Psychological Bulletin, 82:463-496. https: / / doi.org/10.1037/h0076860.

Bickel, W. K., Odum, A. L., and Madden, G. J. (1999). Impulsivity and cigarette smoking: Delay discounting in current, never, and ex-smokers. Psychopharmacology, 146:447-454. https://doi . org/10.1007/pl00005490.

Birk, M., Mandryk, R., Bowey, J., and Buttlar, B. (2015). The effects of adding premise and backstory to psychological tasks. In Researching Gamification: Strategies, Opportunities, Challenges, Ethics. CHI'15 Workshop, Seoul, Korea.

Borges, A. M., Kuang, J., Milhorn, H., and Yi, R. (2016). An alternative approach to calculating area-under-the-curve (auc) in delay discounting research. Journal of the Experimental Analysis of Behavior, 106:145-155. https://doi . org/10 . 1002 / jeab. 219.

Büning, H. (2000). Robustness and power of parametric, nonparametric, robustified, and adaptive tests - the multi-sample location problem. Statistical Papers, 41:381-407. https : / / doi . org/.

Cavallaro, D. (2009). Anime and the visual novel: Narrative structure, design and play at the crossroads of animation and computer games. McFarland.

Cox, D., Klimas, C., Edwards, T. M., Tarrant, D., Graham, S., Akjosch, Chapel, Baccaros, G. C., Mitchell, E., and Skemp, J. (2021). The Twine Cookbook. Version 2.1. Accessed 2021-12-05, https : / / twinery • org/cookbook/.

Cyders, M. A. (2013). Impulsivity and the sexes: Measurement and structural invariance of the UPPS-P Impulsive Behavior Scale. Assessment, 20:86-97. https://doi.org/10.1177/1073191111428762. 
Cyders, M. A., Smith, G. T., Spillane, N. S., Fischer, S., Annus, A. M., and Peterson, C. (2007). Integration of impulsivity and positive mood to predict risky behavior: Development and validation of a measure of positive urgency. Psychological Assessment, 19:107-118. https://doi.org/10.1037/1040-3590.19.1.107.

da Matta, A., Gonçalves, F. L., and Bizarro, L. (2012). Delay discounting: Concepts and measures. Psychology \& Neuroscience, 5:135-146. https://doi.org/10.3922/j.psns.2012.2.03.

DeHart, W. B., Friedel, J. E., Frye, C. C., Galizio, A., and Odum, A. L. (2018). The effects of outcome unit framing on delay discounting. Journal of the Experimental Analysis of Behavior, 110:412-429. https:// doi .org/10.1002/ jeab. 469.

DeHart, W. B. and Odum, A. L. (2015). The effects of the framing of time on delay discounting. Journal of the Experimental Analysis of Behavior, 103:10-21. https://doi.org/10.1002/ jeab.125.

Demetrovics, Z., Urbán, R., Bagygyörgy, K., Farkas, J., Griffiths, M. D., Pápay, O., Kökönyei, G., Felvinczi, K., and Oláh, A. (2012). Development of the problematic online gaming questionnaire (POGQ). PLOS ONE, 7:e36417. https: //doi.org/10.1371/journal.pone.0036417.

DeRight, J. and Jorgensen, R. S. (2015). I just want my research credit: Frequency of suboptimal effort in a non-clinical healthy undergraduate sample. The Clinical Neuropsychologist, 29:101-117. https://doi.org/10.1080/13854046. 2014.989267.

Deterding, S., Dixon, D., Khaled, R., and Nacke, L. (2011). From game design elements to gamefulness: Defining "gamification.". In Proceedings of the 15th international academic MindTrek conference: Envisioning future media environments, pages $9-15$.

Faizal, M. A. (2016). The effects of conversation-gambits visual-novel game on students' english achievement and motivation. In 2016 International Electronics Symposium (IES), pages 481-486.

Franck, C. T., Koffmaus, M. N., House, L. L., and Bickel, W. K. (2015). Accurate characterization of delay discounting: A multiple model approach using approximate bayesian model selection and a unified discounting measure. Journal of the Experimental Analysis of Behavior, 103:218-233. https://doi .org/10.1002/ jeab.128.

Friehs, M. A., Dechant, M., Vedress, S., Frings, C., and Mandryk, R. L. (2020). Effective gamification of the stop-signal task: Two controlled laboratory experiments. JMIR Serious Games, 8:e17810. https://doi.org/10.2196/17810.

Gilroy, S. P. and Hantula, D. A. (2018). Discounting model selection with area-based measures: A case for numerical integration. Journal of the Experimental Analysis of Behavior, 109:433-449. https:// doi .org/10.1002/ jeab.318.

Hakulinen, L., Auvinen, T., and Korhonen, A. (2013). Empirical study on the effect of achievement badges in trakla2 online learning environment. In 2013 Learning and teaching in computing and engineering, pages 47-54. IEEE.

Harman, H., Kodak, T., and McKerchar, T. (2020). Effects of reward magnitude frames on measures of delay discounting in a hypothetical money scenario. Journal of the Experimental Analysis of Behavior, 114:193-202. https://doi .org/10. 1002 / jeab. 620 .

Hommel, G. (1988). A stagewise rejective multiple test procedure based on a mondified bonferroni test. Biometrika, 75:383-386. https://doi.org/10.1093/biomet/75.2.383.

Israel, L. (2017). Creating a video game simulation of alcohol and marijuana use: Pilot study.

Laibson, D. (1997). Golden eggs and hyperbolic discounting. The Quarterly Journal of Economics, 112:443-478. https : //doi.org/10.1162/003355397555253.

Lee, M. D. and Wagenmakers, E.-J. (2013). Bayesian Cognitive Modeling. Cambridge University Press.

Lieberoth, A. (2015). Shallow gamification: Testing psychological effects of framing an activity as a game. Games and Culture, 10:229-248. https://doi.org/10.1177/1555412014559978.

Lumsden, J., Edwards, E. A., Lawrence, N. S., and Munafò, M. R. (2016). Gamification of cognitive assessment and cognitive training: A systematic review of applications and efficacy. JMIR Serious Games, 4:e11. https://doi .org/10.2196/ games. 5888.

Manero, B., Torrente, J., Freire, M., and Fernándex-Manjón, B. (2016). An instrument to build a gamer clustering framework according to gaming preferences and habits. Computers in Human Behavior, 62:353-363. https: // doi . org/.

McAuley, E., Duncan, R., and Tammen, V. V. (1989). Psychometric properties of the intrinsic motivation inventory in a competitive sport setting: A confirmatory factor analysis. Research Quarterly for Exercise and Sport, 60:48-58. https : //doi.org/10.1080/02701367.1989.10607413.

Mitchell, R., Schuster, L., and Jin, H. S. (2020). Gamification and the impact of extrinsic motivation on needs satisfaction: Making work fun? Journal of Business Research, 106:323-330. https://doi.org/10 .1016/j.jbusres . 2018. 11.022.

Myerson, J., Green, L., and Warusawitharana, M. (2001). Area under the curve as a measure of discounting. Journal of the Experimental Analysis of Behavior, 76:235-243. https://doi.org/10.1901/ jeab.2001.76-235.

Odum, A. L. (2011). Delay discounting: I'm a $k$, you're a $k$. Journal of the Experimental Analysis of Behavior, 96:427-439. 
https://doi.org/10.1901/jeab.2011.96-423.

Rachlin, H., Raineri, A., and Cross, D. (1991). Subjective probability and delay. Journal of the Experimental Analysis of Behavior, 55:233-244. https://doi.org/10.1901/ jeab.1991.55-233.

Reynolds, B. and Schiffbauer, R. (2004). Measuring state changes in human delay discounting: An experiential discounting task. Behavioural Processes, 67:343-356. https:// doi .org/10.1016/j . beproc.2004.06.003.

Robles, E. and Vargas, P. A. (2007). Functional parameters of delay discounting assessment tasks: Order of presentation. Behavioural Processes, 75:237-241. https://doi.org/10.1016/j.beproc.2007.02.014.

Schluter, M. G., Kim, H. S., and Hodgins, D. C. (2018). Obtaining quality data using behavioral measures of impulsivity in gambling research with amazon's mechanical turk. Journal of Behavioral Addictions, 7:1122-1131. https: / / do . org/ $10.1556 / 2006.7 .2018 .117$.

Team, S. D. (2021). Stan Modeling Language Users Guide and Reference Manual. Version 2.28. https : / / mc-stan . org. Vincent, B. T. (2016). Hierarchical Bayesian estimation and hypothesis testing for delay discounting tasks. Behavior Research Methods, 48:1608-1620. https: / / doi.org/10.3758/s13428-015-0672-2.

Weinstein, A., Abu, H. B., Timor, A., and Mama, Y. (2016). Delay discounting, risk-taking, and rejection sensitivity among individuals with internet and video gaming disorders. Journal of Behavioral Addictions, 5:674-682. https: //doi.org/10.1556/2006.5.2016.081.

Werbach, K. (2014). (Re) defining gamification: A process approach. In International conference on persuasive technology, pages 266-272. Springer.

Wulff, D. U. and van den Bos, W. (2018). Modeling choices in delay discounting. Psychological Science, 29:1890-1894. https://doi.org/10.1177/0956797616664342. 